\title{
Community-Acquired Pneumonia (International Vision)
}

\author{
Alejandro RodríGueZ AND Jordi Rello
}

\begin{abstract}
Community-acquired pneumonia (CAP) is generally regarded as pneumonia requiring a specific clinical approach in the intensive care unit (ICU). Between $10 \%$ and $36 \%$ of patients with CAP who are hospitalized need ICU treatment, and mortality ranges from $20 \%$ to $50 \%$. The ICU admission and management should be customized in each patient.

The spectrum of causative agents of severe CAP (SCAP) is similar to that found in hospitalized patients or outpatients with CAP. Streptococcus pneumoniae and Legionella species (spp.) account for 50\% of cases with etiologic diagnosis. However, Legionella spp. are rarely reported in South America, where S. pneumoniae, Haemophilus influenzae, and Staphylococcus aureus are the most frequent findings. Interestingly, ICU admission for tuberculosis can reach $10 \%$ of total cases in some countries.

The usefulness of diagnostic testing in the management of SCAP is a subject of controversy. However, knowledge of the causative agents is useful, given the possibility of adjusting antibiotic treatment, and improves the outcome. Blood culture, Gram stain, and urinary detection of antigens should be done.

Shock and refractory hypoxemia management do not differ for the general population with septic shock. The goal of resuscitation in shock is the restoration of tissue perfusion. Normalization of cellular metabolism and noninvasive forms of ventilation or a protective ventilatory strategy using low tidal volumes are recommended.

The guidelines for initial antibiotic management should be customized to each country or to each institution and patient. We believe that cefotaxime/ ceftriaxone should be the cornerstone of therapy in combination with macrolide, but antibiotic coverage for Legionella spp. and Pseudomonas aeruginosa might be necessary in intubated critically ill patients when SCAP etiology is unknown.
\end{abstract}




\section{Introduction}

Community-acquired pneumonia (CAP) is among the most common infectious diseases, although the precise figures are not available for most countries, because appropriate studies have not been performed. CAP accounts for more than 600,000 hospital admissions in the United States ${ }^{1}$ and for more than 50,000 in the United Kingdom each year. ${ }^{2}$ Studies in Spain ${ }^{3}$ and Finland suggested frequencies of 2.6 and 4.7 per 1,000 in the general population per year. The frequency of the condition is country related and age related, with the highest rates in the extreme of life. Its frequency, costs, morbidity, and mortality (20\% to $50 \%$ ) are the reasons CAP is such an important disease. ${ }^{4-6}$

The steady increase in the number of senior citizens and immunocompromised patients, the better survival rates of patients affected by chronic illness, and the necessary administration of appropriate empirical antibiotics (ATB) justify continuing research in this field. In the last decade, several medical societies published guidelines for the initial CAP management for attempts to reduce the high mortality. ${ }^{7-9}$ The clinical utility, acceptance and use for the physician, and impact on the outcome of such guidelines are unknown.

This chapter focuses on the subgroup of CAP patients that are admitted to the ICU (approximately $10 \%$ of patients hospitalized) and reviews the most important factors regarding etiology, prognosis, diagnostic tools, and treatment. Finally, we develop an algorithm of diagnosis and treatment (Figure 3.1).

\section{Definition}

CAP is defined as an acute lower respiratory tract infection characterized by the following: (a) an acute pulmonary infiltrate evident on the chest radiographs and compatible with pneumonia, (b) confirmatory clinical examination, and (c) acquisition of the infection outside the confines of the hospital, chronic care facility, or nursing home. Pneumonia is characterized pathologically by inflammation within and around the alveolar spaces (lung consolidation) with disruptions of the normal gas exchange and clinical symptoms of dyspnea, cough, and expectoration. ${ }^{8,9}$

However, no consensus has been reached among researchers with regard to the definition of severe pneumonia. Obviously, the condition of patients admitted to the ICU for control of vital constants, shock correction, or mechanical ventilation can be considered severe. but criteria for ICU admission and the infrastructure may differ from hospital to hospital. The American Thoracic Society (ATS) guidelines, ${ }^{7}$ recently validated, suggested 10 items for definition of severity (Table 3.1). However, Ewing, et al. ${ }^{10}$ found that the presence of at least one of 10 criteria had high sensitivity (98\%) but low 


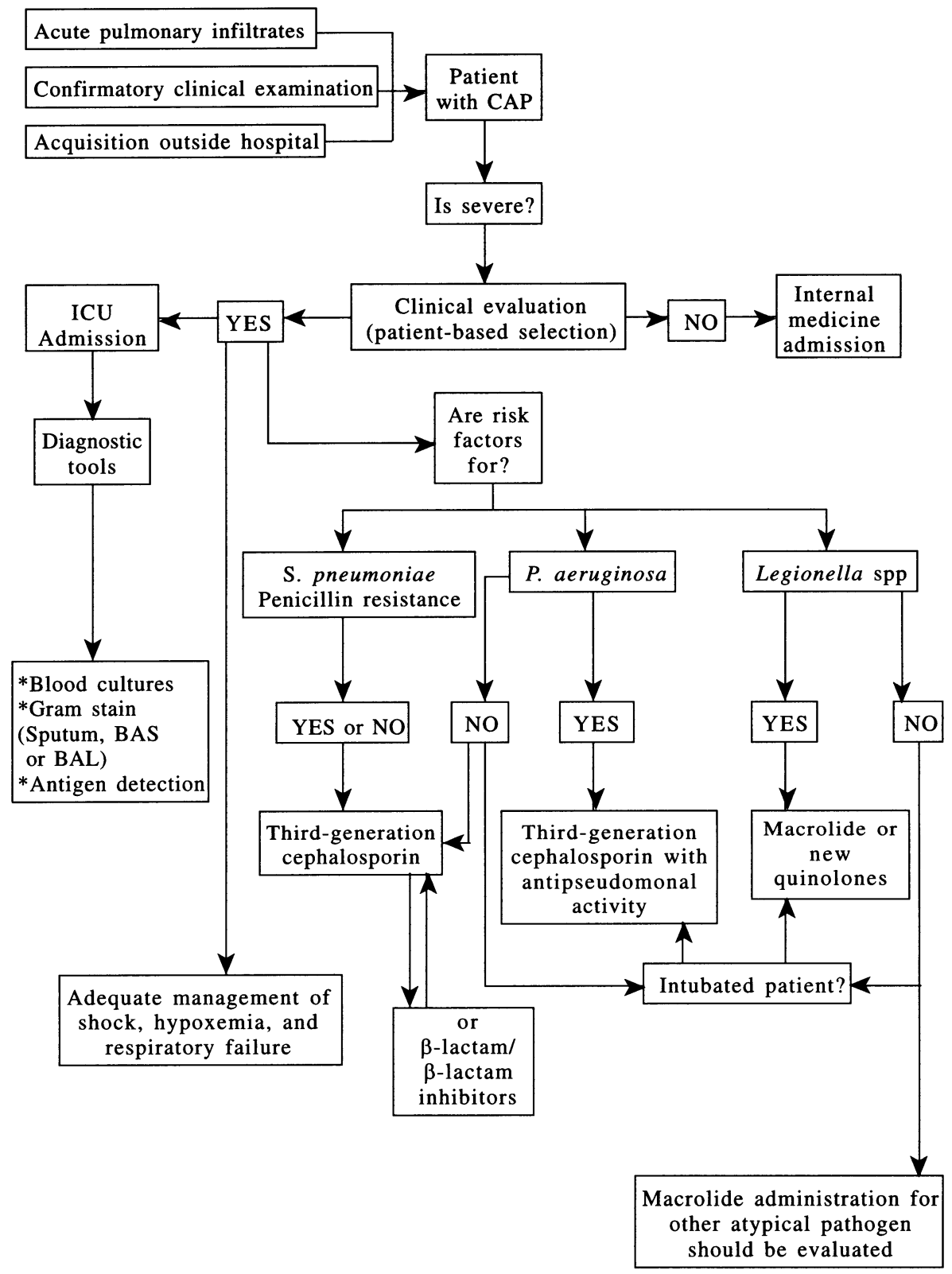

Figure 3.1. Diagnostic and treatment algorithm for severe CAP. 
Table 3.1. ATS criteria of severity in CAP

- Respiratory rate $>30 / \mathrm{min}$

- Severe respiratory failure $\left(\mathrm{Pa}_{\mathrm{O} 2} / \mathrm{Fi}_{\mathrm{O} 2}<250\right)$

- Requirement for mechanical ventilation

- Bilateral involvement in chest radiograph

- Increase in the size of the opacity by $50 \%$ or greater within 48 hours

- Systolic blood pressure $<90 \mathrm{mmHg}$

- Diastolic blood pressure $<60 \mathrm{mmHg}$

- Requirement of vasopresors $>4$ hours

- Urine output lower than $20 \mathrm{~mL} / \mathrm{h}$, or total urine output lower than $80 \mathrm{~mL}$ in 4 hours

- Acute renal failure requiring dialysis

specificity (32\%). The authors suggested that the definition of severity would need one "major" criterion (requirement of vasopressors of more than 4 hours or mechanical ventilation) or at least two "minor" criteria (systolic blood pressure of less than $90 \mathrm{mmHg}, \mathrm{PaO}_{2} / \mathrm{FiO}_{2}$ of less than $250 \mathrm{mmHg}$, involvement of more than two lobes in chest radiograph).

Using multivariate analysis, the British Thoracic Society (BTS) ${ }^{11}$ concluded that only three variables were associated with an increased risk of mortality: respiratory rate of more than 30 breaths $/ \mathrm{min}$, blood urea of more than $7 \mathrm{mmol} /$ $\mathrm{L}$, and diastolic blood pressure of less than $60 \mathrm{mmHg}$. The association of at least two of these variables increased the mortality risk 21 times.

A recent study ${ }^{12}$ reports that the pneumonia severity index (PSI) ${ }^{13}$ has a poor positive predictive value of $16 \%$. These findings agree with previous reports, which find that inappropriate hospitalization rates were $5 \%{ }^{14}$ and $23 \% .{ }^{15}$ We believe that the decision with regard to ICU admission must be individualized (patient-based selection) rather than based on a severity index. It should be based on the stability of the patient's clinical condition, the risk of death or complications, and the presence of other active medical problems.

\section{Etiology and Risk Factors}

The spectrum of causative agents of SCAP is similar to that found in hospitalized patients or outpatients with CAP. However, a number of issues may confound the results of etiologic studies in CAP. Health care factors (criteria for ICU admission, proportion of patients admitted to hospital) and several factors related to the population being studied (number of patients studied, the age mix, antibiotic therapy, etc.) may have an impact on etiologic results.

Table 3.2 shows that S. pneumoniae and Legionella spp. account for $50 \%$ of cases with etiologic diagnosis. Interestingly, in some countries, such as those of South America, Legionella spp. are rarely reported, and S. pneumoniae, 


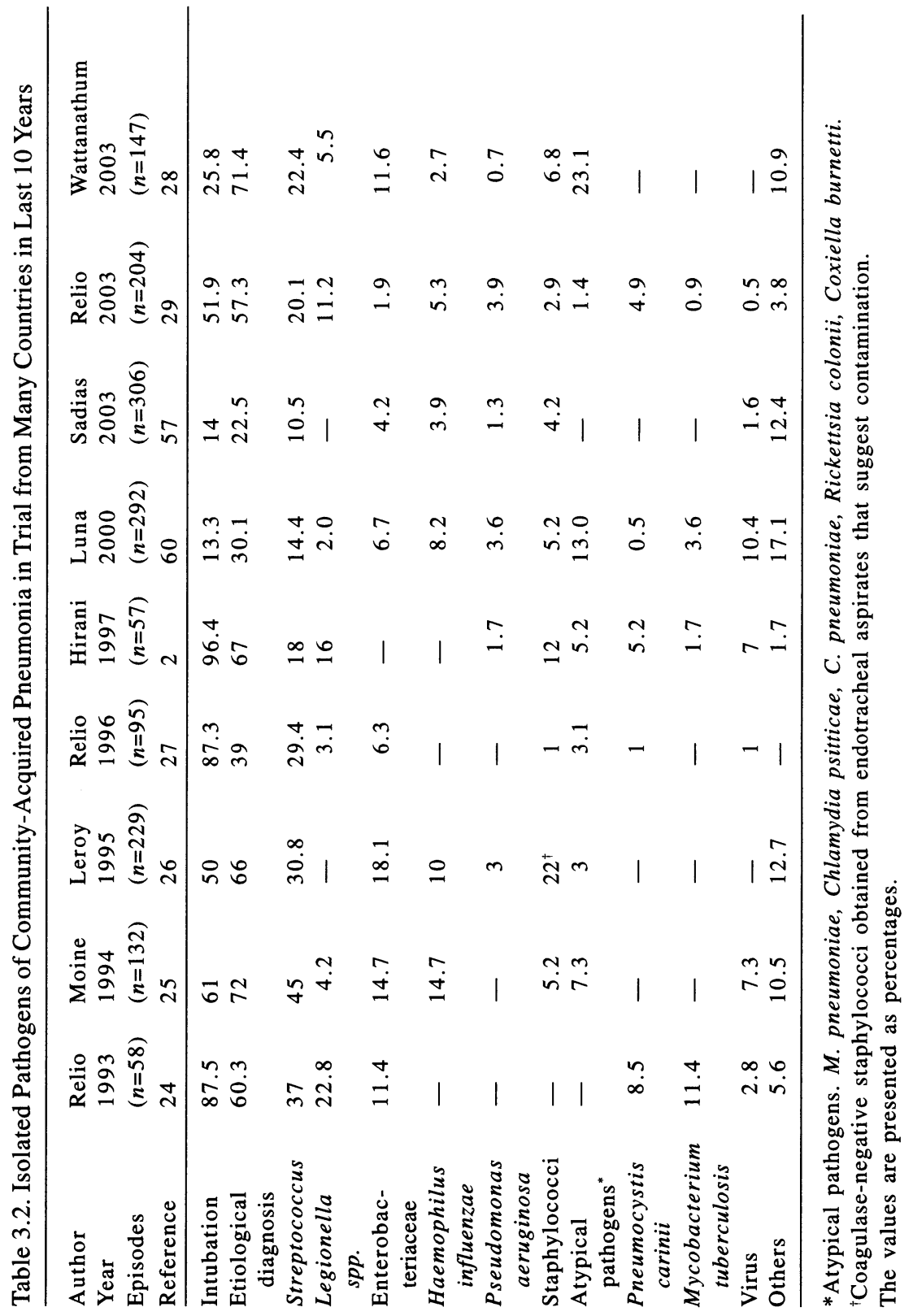


Haemophilus influenzae, and $S$. aureus are the most frequent findings. In developing countries, ICU admission for tuberculosis can reach $10 \%$ of total cases.

The most important risk factors for $S$. pneumoniae infection are chronic hepatic disease, alcoholism, influenza, cigarette smoking, and chronic obstructive pulmonary disease (COPD). ${ }^{6}$ A recent Spanish multicenter study in elderly patients ${ }^{16}$ found $S$. pneumoniae in $49 \%$ of patients in isolation. The impact of age appears to be mainly on the frequency of pneumococcal infection, which is more common in the elderly. In patients with human immunodeficiency virus (HIV) infection, the risk of pneumococcal infection correlates with the CD4 count (below 200 significantly increases the risk of pneumococcal pneumonia). ${ }^{6}$

The most important risk factors for Legionella spp. infection are advanced age, smoking, steroid therapy, COPD, alcoholism, and other forms of immunosuppressive therapy. ${ }^{6,17-19}$ Legionella spp. are an uncommon cause in the hospital, but infection from this source in previously healthy patients has been reported, even in case of nosocomial acquisition. ${ }^{6}$ Legionnaires disease may occur in previously healthy subjects, and the absence of underlying disease should not be a reason for excluding the diagnosis. ${ }^{18}$ On the other hand, nosocomial Legionnaires disease may occur in epidemics, but the detection of most such outbreaks usually infers the existence of prior unsuspected sporadic nosocomial cases ${ }^{18,19}$

In South America, Legionella pneumonia is rarely found. Lopardo et al. ${ }^{20}$ studied the incidence of acute infection due to Legionella spp. in a group of adult patients in Buenos Aires. Ninety-two of the enrolled patients could be evaluated. Only three patients $(3.3 \%)$ fulfilled the serologic diagnostic criteria for Legionella spp. acute infection. Recently, eight patients were reported in Chile. ${ }^{21}$ Both series made the etiologic diagnosis just by urinary antigen or serologic test. No diagnoses were confirmed by microorganism cultures, and the mortality was extremely low. There is a perception that the Legionella organism occurs more often in Mediterranean countries and in the United States and is less frequent in northern European and South American countries. ${ }^{6-22}$

$H$. influenzae is the third most common cause of CAP requiring hospitalization and accounts for between $6 \%$ and $15 \%{ }^{23-28}$ of pneumonia that requires ICU admission. COPD, elderly, and HIV patients are mainly affected.

$S$. aureus and Gram-negative enteric bacteria are uncommon in disease managed outside a hospital, but infection from these sources shows a pattern of progressive increase in frequency with increasing severity. ${ }^{22}$ Pneumonia caused by $S$. aureus is usually a severe infection, requiring ventilatory support in up to $90 \%$ of cases. ${ }^{6}$ The infection occurs after epidemic influenza or via the bloodstream.

Among Gram-negative bacteria, $P$. aeruginosa stands out on account of its extreme virulence. Fortunately, the incidence in CAP is low, and this etiology should be considered in patients with structural lung disease and patients with neutropenia. However, in a recent study ${ }^{29}$ in patients admitted to the ICU 
with SCAP our group found $P$. aeruginosa in only eight patients (3.9\%), but seven patients died. Interestingly, $P$. aeruginosa was fourth in the ranking of lethal pathogens in intubated patients and was not predicted by the presence of comorbidities. ${ }^{29}$ This finding suggests that empiric therapy for SCAP in intubated patients should be active against this pathogen because a delay in therapy is associated with increased mortality. ${ }^{29}$

Chlamydia pneumoniae is one of the least well understood organisms as it has been detected in the most recent studies. ${ }^{22}$ In South America the incidence of $C$. pneumoniae and Mycoplasma pneumoniae was reported at about $10 \% .{ }^{30-32} \mathrm{~A}$ recent prospective cohort study ${ }^{33}$ in Canada reported that only $2.2 \%(12 / 539)$ of patients have acute $C$. pneumoniae pneumonia and an additional 5.9\% (32/539) had possible acute infection. Interestingly, in only $38 \%$ patients, $C$. pneumoniae was the sole pathogen finding. In $62 \%$ of cases, this microorganism was isolatfed as a co-pathogen to $S$. pneumoniae and respiratory syncytial or influenza virus. These results suggest that $C$. pneumoniae is an important cause of CAP, but the true role in SCAP is uncertain.

Finally, in a high percentage of patients (30\% to $50 \%$ ) the causative agent is impossible to determine. The frequency of this situation varies among studies for several reasons, depending on diagnostic methods. Recently, Menéndez et al., ${ }^{34}$ using polymerase chain reaction (PCR) techniques, confirmed that in patients with unknown etiology, $S$. pneumoniae was the most probable cause.

\section{Diagnosis}

The usefulness of diagnostic testing in the management of CAP is a subject of controversy. ${ }^{35}$ Therefore, the empiricism is the usual approach in many patients with CAP. Indeed, some guidelines strongly recommend it. ${ }^{7}$ Several previous reports ${ }^{36,37}$ have evaluated the impact of diagnostic testing in the population hospitalized in medical wards. They state that knowledge of the pathogen may not affect clinical outcome, concluding that routine microbiological investigation is unhelpful and is probably unnecessary. However, knowledge of the causative agent is useful, given the possibility of adjusting antibiotic treatment. In our experience ${ }^{29}$ the result of microbiologic investigation in the clinical setting of critically ill patients led to a change in therapy in approximately $40 \%$ of cases. Moreover, the identification of the microorganism after the initial incorrect empirical therapy and subsequent correction of treatment to cover the offending pathogen was followed by clinical resolution in seven of 11 patients. In addition, performing microbiologic studies led to simplification of treatment in one-third of patients, reducing antibiotic expense, helping to reduce side effects, and preventing antibiotic abuse.

At clinical practice, in SCAP, we recommend obtaining at least two blood cultures and respiratory samples for culture and Gram stain. However, it is extremely difficult (only $20 \%$ ) to obtain a good sputum sample (more than 25 
Table 3.3. Advantages/Disadvantages of the Sputum Gram Stain

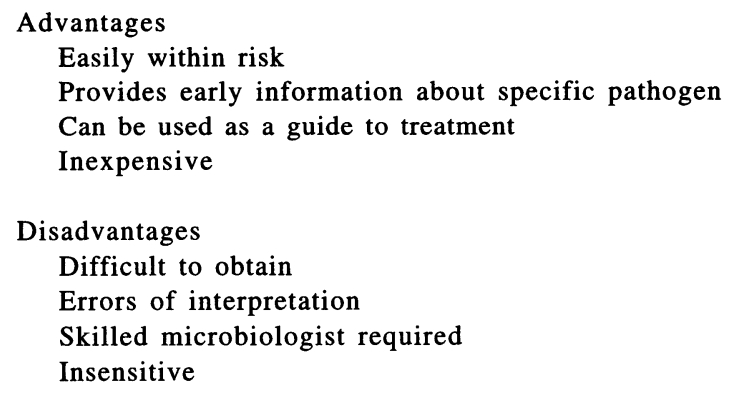

Modified from Jasovich A, Soutric J, Morera G, et al. Efficacy of amoxicillin-sulbactam, given twice a day for the treatment of community-acquire pneumonia. A clinical trial based on a pharmacodynamic model. J Chemother 2002;14:591-6.

neutrophils and fewer than 10 epithelial cells per microscopic field $(<t s>100))$ for Gram stain from these patients. ${ }^{38}$ In addition to the difficulty of obtaining the sample, the interpretation of the results is complicated and depends on the diagnostic criteria used, and a skilled interpreter ${ }^{39}$ Table 3.3 shows the advantages and disadvantages of the sputum Gram stain in the diagnosis of CAP. The proper interpretation of Gram stain can be affected by staff training and previous use of antibiotics. Indeed, previous antibiotic use may sterilize cultures, particularly in cases due to $H$. influenzae and $S$. pneumoniae ${ }^{6}$ However, the sputum induced by hypertonic saline solution has proved to be a good tool for Pneumocystis carinii and tuberculosis investigation, especially in AIDS.

In many countries of South America the sputum Gram stain is frequently of limited value because many patients receive antibiotics before admission (self-medication) and a skilled microbiologist is not always available.

Blood cultures (BC) are considered a standard of care for patients hospitalized for CAP. They are positive in around $10 \%$ to $30 \%$ of patients with CAP ${ }^{6,40}$ and are included in all major contemporary guidelines for CAP management. ${ }^{1,41}$ However, the clinical value of routine $\mathrm{BC}$ has been questioned. ${ }^{42,43}$ Recently, Campbell et al. ${ }^{43}$ found that $\mathrm{BC}$ have limited usefulness in the routine management of patients with CAP. Sanyal et al ${ }^{42}$ found no difference in mortality between patients in whom antibiotics were changed empirically $(64 \%)$ and in those who experienced change as a result of positive microbiologic study $(67 \%)$. Similar findings reported by other authors ${ }^{37}$ indicated a mortality of $16 \%$ in patients with blind change in antibiotic therapy versus $25 \%$ in those who underwent microbiologically guided change. The blood culture dates did not have a significant impact in directing antibiotic changes. ${ }^{36}$ In this study, 93 patients admitted with SCAP, of whom 69 (74\%) had failed initial empirical antibiotic therapy, showed little benefit from microbiologic studies. 
In these studies, the antibiotic therapy was changed very much later. Initial appropriate empiric antibiotic therapy is critical, particularly in critically ill patients. ${ }^{24,29,44}$ The late confirmation of bacteremia may occasionally be of help to verify retrospectively the diagnosis in patients with SCAP, but a late change in antibiotic therapy does not improve the outcome. The identification of a microorganism not covered by initial empiric therapy will not have a beneficial effect on survival within the first days after admission. However, our group ${ }^{45}$ showed that an early change (less than 48 hours) in the therapy guided by culture dates from samples obtained within the first 12 hours after admission permitted rescue and clinical resolution in two-thirds of patients.

A prospective study of patients with $\mathrm{CAP}^{39}$ showed that the yield of blood culture increases significantly with the severity of pneumonia and that positive cultures only impacted on the management of critically ill patients (PSI IV and V). The ICU patients with SCAP have these levels of severity. We believe that despite low sensitivity of blood culture, the convincing nature of the isolation of a respiratory pathogen from blood, the opportunity to test the antimicrobial sensitivity of the isolate, and the relative simplicity of drawing blood for cultures (two samples) all argue in favor of obtaining blood cultures in patients requiring ICU admission.

Noninvasive techniques are based on antigen detection of some microorganisms in urine, plasma, or sputum. The most useful is antigen detection of Legionella spp. in urine. Sensitivity is around $90 \%$, and specificity is nearly $100 \% .^{18}$ The main drawback is that only serogroup 1 can be detected, although serogroups account for more than $70 \%$ of Legionella infection. A negative urinary antigen test does not rule out legionellosis, but a positive test is highly specific. In both the $\mathrm{ATS}^{46}$ and the Canadian guidelines ${ }^{41}$ the urine test for Legionella spp. is recommended in patients with CAP. This test is not influenced by previous use of antibiotic and can remain positive for a long time after pulmonary infection. Currently, techniques based on PCR and latex agglutination are available, especially in the case of the Pneumococcus. Procedures based on PCR techniques can be applied to noninvasive samples. Sensitivity remains high despite antibiotic treatment. In addition, the results are available within only a few hours. However, the high cost of these procedures and the lack of well-prepared laboratories preclude their worldwide use.

Other useful diagnostic tools are based on invasive tests. There is considerable controversy about the role and use of invasive testing in the diagnosis of CAP. The use of bronchoscopy for patients with CAP who had failed initial management has identified an infectious agent in fewer than $30 \%$ of patients. ${ }^{35,47}$ Bronchoscopy is easy to perform when a patient is intubated, but it tends to be little used because the necessary laboratory equipment and welltrained bronchoscopy staff are frequently not available. Furthermore, in nonintubated patients, respiratory failure constitutes a relative contraindication, given the possibility of speeding up urgent intubation. In the most recent guidelines for CAP management, ${ }^{8,41,46}$ bronchoscopic sampling is recommended for particularly severe, selected cases but not for all patients un- 
der mechanical ventilation. The few studies undertaken in SCAP have reported that the reliability of bronchoscopy is high. However, in immunocompromised patients (those who have HIV infection, are under steroidal therapy, or have had a transplant) or in those with a high suspicion of atypical microorganisms, bronchoscopy with bronchoalveolar lavage (BAL) is the first step in the diagnostic approach. The availability of a skilled bronchoscopist and an experienced microbiologist is a key factor in the decision of whether to perform invasive sampling on ICU admission.

\section{Mortality and Associated Factors}

Despite advances in antibiotic treatments and technical improvements in ICU care, SCAP mortality remains unacceptably high. In different studies of SCAP that required ICU admission, crude mortality is around $20 \%$ to $54 \% .{ }^{23,24,29,40} \mathrm{~A}$ recent metaanalysis by Fine et al. ${ }^{48}$ confirmed these data on mortality. In that study 33,148 patients were evaluated from 127 publications. Thirteen of these studies were of patients admitted to the ICU. Mortality in hospitalized patients was $14 \%$, and was $36.5 \%$ among those admitted to the ICU. Several factors have been associated with poor outcome.

Age has been considered for a long time as a risk factor. A study from Canada by Marrie et al. ${ }^{49}$ in a population older than 65 concluded that the mortality rate increased with age, reaching $61 \%$ in those more than 71 years of age. In a casecontrol study (those more than 65 years of age) performed in 101 pneumonia patients, Riquelme et al. ${ }^{50}$ concluded that the risk factors of CAP in this group were gastric aspiration and malnutrition. The two groups were similar with relation to age, gender, and day of admittance. The significant prognostic factors were bedridden patients (relative risk-RR, 10.7), deglutition impairment ( $R R$, $7.3)$, lack of fever (RR, 10.5), respiratory rate in excess of $30 / \mathrm{min}(R R, 5.2)$, and bilateral and multilobar (more than three lobules) involvement in chest radiography $(R R, 2.3)$. Recently, Fernandez-Sabe et al. ${ }^{51}$ observed that early mortality and overall mortality were significantly higher in very elderly patients (those more than 80 years of age). The factors independently associated with 30-day mortality were altered mental status on admission (Odds Ratio-OR, 3.6), shock (OR, 10.6), respiratory failure $(\mathrm{OR}, 3.5)$, renal failure $(\mathrm{OR}, 5.8)$, and Gram-negative pneumonia (OR, 20.2). Finally, age of more than 65 years $(\mathrm{OR}, 3.5)$ and albumin of less than $30 \mathrm{~g} / \mathrm{dL}(\mathrm{OR}, 4.7)$ were factors independently associated with the mortality for Lim et al. ${ }^{52}$

However, age itself was not a factor that indicated a bad prognosis. The microbial virulence, bacterial burden, and patients' underlying diseases are very important prognostic factors. In a prospective study performed in COPD patients admitted for CAP, ${ }^{53}$ mortality was only $23 \%$ in the 22 patients who required ICU admission. This percentage of mortality is similar to the rate in COPD patients undergoing intubation for exacerbation. In a multicenter study performed in four ICUs ${ }^{44}$ crude mortality in exacerbated COPD patients was 
$24 \%$. This level of mortality was not related to COPD status but to multiorgan failure. However, when mortality was evaluated at 180 days, underlying disease was clearly related to mortality. Severe COPD patients had fewer possibilities of survival than less severe COPD patients. Finally, in the metaanalysis by Fine et al., ${ }^{48}$ neither COPD nor alcoholism were among the 11 prognostic significant factors. ${ }^{54}$ These results agree with our findings in elderly patients ${ }^{24}$ In that study, comorbidities were not related to pneumonia outcome, excepting immunosuppression.

\section{Impact of the Use of Guidelines on Mortality}

Several studies using multivariate analysis have shown that inadequate antibiotic treatment is associated with a significant increase in mortality. ${ }^{23,24,29,55}$ In a multicenter, retrospective study ${ }^{56}$ of more than 14,000 patients older than 65 years with CAP, Meehan et al. reported that delaying the start of antibiotic treatment by more 8 hours and failure to perform blood cultures within the first 24 hours were associated with increased mortality at 30 days of admission. In spite of these findings, other studies that specifically addressed SCAP in the ICU reported that the correct adherence to guidelines did not increase survival. $^{2,57}$ In these studies ${ }^{2}$ of 57 SCAP patients, the management guidelines were adopted but without a reduction in mortality. The mortality was $58 \%$ versus $54 \%$ prior to ATS guidelines. In Chile, Saldías Peñafiel et al. ${ }^{57}$ reported that the use of macrolides combined with â-lactams had no effect on clinical course or prognosis in patients with CAP. Considering the low prevalence of multiresistant pneumococci and the low incidence of atypical pathogens in many countries of South America, no clinical and/or bacteriologic basis exists for using foreign guidelines.

Therefore no simple set of guidelines is applicable to every patient. Ultimately, treatment decision depends on good clinical judgment and must take into account patient demographics, local antibiotic resistance patterns, and epidemiologic data. A great variability may be noted among different countries in the selection of first-choice antimicrobial agents. This may be because of many microbial causes of CAP and specialties involved, as well as different health-care systems, which affect the availability or cost of antibiotics. Therefore, each country should develop local evidence-based guidelines for the initial management of CAP. Recently, in South America a committee of physicians with a strong interest in CAP recommended initial antimicrobial treatment of CAP on the basis of local evidence. ${ }^{58}$

\section{Antibiotic Treatment}

Usually, antibiotic treatment is started empirically, trying to cover the most frequent microorganisms and taking into account the risk factors for specific agents. For accurate treatment, it is critical to identify whether pulmonary 
infection is due to $P$. aeruginosa, Legionella, or penicillin-resistant pneumococci (PRSP). Given that clinical differentiation is usually inaccurate, most guidelines recommend expanded-spectrum antibiotic therapy. A combination of two antibiotics is generally preferred.

$P$. aeruginosa is a possible agent in CAP only in certain settings and requires specific therapy. The 2001 update of the ATS guidelines ${ }^{46}$ recommends that this pathogen be considered only when specific risk factors (bronchiectasis) are present. Our group ${ }^{29}$ reported that patients with SCAP who underwent intubation were exposed to significant risk of infection by relatively infrequent but highly lethal pathogens, such as Legionella or Pseudomonas spp. We suggest that both pathogens be covered (in addition to the pneumococcus, of course) in the empiric therapy of all intubated patients while awaiting bacteriology results.

In the IDSA guidelines ${ }^{1}$ for ICU patients, third-generation cephalosporin such as cefotaxime or ceftriaxone (or $\beta$-lactam $\beta$-lactamase inhibitors) plus macrolide or fluoroquinolones are recommended. The use of macrolides (or new quinolones) is due to the high incidence of Legionella infection. In a recent study, our group ${ }^{59}$ evaluated the different antibiotic choices and their relation to outcome in patients with SCAP. The mortality was similar in patients receiving a macrolide alone $(18 \%)$ and in those who received it in combination with $\beta$-lactam $(27 \%)$. Interestingly, we found an excess mortality $(14 \%)$ for initial treatment with an aminoglycoside alone or with another agent. In addition, no different mortality was found between monotherapy or combination therapy. However, these results should bereevaluated for South America, where the prevalence of PRSP and legionellosis is low. ${ }^{20,21,60}$

In some geographic areas PRSP has become an important problem. Data for invasive isolates collected in 2001 by European Antimicrobial Resistance Surveillance System (EARSS) identifies Spain and Greece as having the highest (more than $30 \%$ of isolates) frequencies of penicillin resistance, followed by $10 \%$ to $29 \%$ in Belgium, Poland, Hungary, and Slovenia. ${ }^{22}$ The association between penicillin resistance and adverse clinical outcome has been well documented for meningitis but is not well defined for pneumonia. ${ }^{35}$ Despite some reports of poor outcome among patients infected with intermediatesusceptibility strains, most current available evidence indicates that standard treatment with adequate doses of $\beta$-lactams is effective against pneumococcal pneumonia caused by strains with minimal inhibitory concentration (MIC) of less than $1 \mu \mathrm{g} / \mathrm{mL}$. A recent study ${ }^{61}$ of 100 consecutive patients with bacteremia pneumococcal pneumonia identified discordant therapy using the 2002 National Committee for Chemical Laboratory Standards (NCCLS) breakpoints as a significant variable associated with mortality (OR, 27). Using cefotaxime or ceftriaxone, the risk of discordant therapy is only $2 \%$. Cefuroxime should not be used because it has a significant association with mortality. ${ }^{62}$

In South America a study by Artemis, carried out from 1997 to 1998, analyzed 600 strains of $S$. pneumoniae. This study found that $18.5 \%$ patients were resistant to penicillin. Nearly half of these cases were highly resistant (minimal inhibitory concentration MIC >2) ${ }^{63}$ Other studies confirmed these 
data with $20 \%$ to $25 \%$ of PRSP. ${ }^{64}$ In a recent international study ${ }^{63}$ the incidence of PRSP in Argentina was $2.6 \%$ and $18.7 \%$ in Brazil. These results agree with other studies in Argentina, specifically designed to compare the frequency of PRSP. ${ }^{58}$ In 121 patients with CAP only two (4.4\%) S. pneumoniae strains with decreased susceptibility to penicillin (MIC 0.25 to $1 \mu \mathrm{g} / \mathrm{mL}$ ) were found among 45 isolates recovered. On the basis of the current evidence, the clinical impact of the PRSP on CAP management in South America is poor.start here

Because of the worldwide increase in PRSP and the high incidence of Legionella spp. infection in SCAP (not in South America), newer antibiotics have been developed to cover both microorganisms. Newer fluoroquinolones (levofloxacin, grepafloxacin, moxifloxacin, and trovafloxacin) seem to offer good coverage against PRSP and Legionella, and excellent lung tissue levels have been reported. These antibiotics might be a good alternative for CAP patients with â-lactam hypersensitivity. Unfortunately, no clinical trials in patients with SCAP are available, and their use in critically ill patients is not supported by evidence.

\section{Shock Management}

Hemodynamic instability is defined by the need for vasoactive drugs, once the hypovolemic state has been corrected, in order to achieve a mean arterial blood pressure (MAP) sufficient to preserve peripheral perfusion. About $25 \%$ to $50 \%$ of patients with CAP have shock septic criteria when admitted to the ICU.

The specific management of patients with SCAP and shock does not differ from that of the general population with septic shock. ${ }^{65}$ The volume repletion in patients with septic shock produces increases in cardiac output and oxygen delivery, and generally is enough to reverse hypotension. ${ }^{66}$ However, the requirements of volume are not easily determined in critically ill patients. We believe that central venous pressure (CVP) is initially required to assess intravascular volume. If CVP increases or the patient develops respiratory failure and requires mechanical ventilation, a pulmonary artery catheter is necessary before administration of vasoactive drugs. In addition, patients should have an arterial catheter in place for continuous blood pressure monitoring and withdrawal of blood samples.

Dopamine is the first approach to patients with septic shock. It increases mean arterial pressure (MAP) primarily by increasing cardiac index. High doses of dopamine increase systemic vascular resistance and might impair splanchnic blood flow. ${ }^{67}$ We suggest that the dopamine doses not exceed 10 $\mathrm{mg} / \mathrm{kg} / \mathrm{min}$, and norepinephrine should be administered (alone or with dopamine) to improve MAP.

Norepinephrine is probably more effective than dopamine at reversing hypotension in septic shock patients. Norepinephrine usually causes a clinically significant increase in MAP (vasoconstrictive effects) with little change 
in heart rate or cardiac output. ${ }^{68}$ In addition, it significantly improves glomerular filtration, and this is particularly true in hyperdynamic septic shock. ${ }^{66}$ We believe that norepinephrine should be used early in septic shock and not as a final tool.

The precise level of MAP is not entirely certain and is likely to vary among patients. Assessment of regional and global perfusion should be evaluated by mixed venous oxygen saturation, ${ }^{69}$ blood lactate levels $(>2 \mathrm{mEq} / \mathrm{L})^{70}$ or gut tonometry ${ }^{71}$ to guide optimum reanimation. We believe that the goal of resuscitation in shock is the restoration of tissue perfusion and normalization of cellular metabolism and not simply to reverse the hypotension.

\section{Treatment of Refractory Hypoxemia}

Among patients admitted to the ICU for SCAP, between $58 \%$ and $88 \%$ need mechanical ventilation. ${ }^{6,38}$ The goal in these patients is to improve gas interchange, keeping plateau pressure low $(<30 \mathrm{mmHg})$ in order to avoid acute lung injury.

The main drawback of intubation and invasive mechanical ventilation is that it increases the probability of superinfections. New forms of ventilation without intubation have been promoted in recent years. ${ }^{72,73}$ Noninvasive forms of ventilation (NIV) have been tested in several diseases and are very useful in COPD patients. In SCAP patients, Confalonieri et al. ${ }^{74}$ reported that NIV was associated with a significant reduction in the rate of endotracheal intubation and duration of ICU stay. In addition, a significant reduction of mortality was observed in COPD patients. On the order hand, despite initial improvement in arterial oxygenation with NIV in patients non-COPD with SCAP, the intubation rate was high $(66 \%) .{ }^{75}$ However, the more favorable outcome and shorter ICU stays were observed when intubation was avoided.

In general, our policy is to implement NIV whenever possible to avoid endotracheal intubation. When the level of consciousness is depressed or the ability to clear secretions is impaired, we avoid their use. The success of this approach depends mainly on training of nurses and respiratory therapists and the nature (severity) of the acute lung injury.

SCAP is associated with acute respiratory distress syndrome (ARDS) in about $10 \%$ of cases. ${ }^{38}$ When ARDS is developed, mechanical ventilation is needed, requiring high levels of oxygen delivery as well as high levels of PEEP to ensure arterial oxygen saturation of $90 \%$. Two goals in this context are the maintenance of low $\mathrm{FiO}_{2}$ and tidal volume. In general, it is desirable to achieve a level PEEP that maintains $\mathrm{FiO}_{2}$ below 0.6 whenever possible. A protective ventilatory strategy using low tidal volumes improves survival in patients with ARDS. ${ }^{76}$ Alveolar recruitment (open lung therapy) maneuvers and placement of patients in a prone position as early rescue adjunctive therapy for severe hypoxemia have been tested. 


\section{Conclusion}

SCAP is an important challenge for clinicians, because of the high rate of mortality despite the use of new antibiotics and sophisticated technology in the ICU setting. ICU admission and management show important divergences regarding clinical practices and should be tailored for each patient. The guidelines for initial management should be adjusted for each country or each institution and patient. Prognostic scoring rules are adjunctive tools but should not be applied to individual patients to guide ICU admission. Although risk factors of mortality are well known, pneumonia is a dynamic process that needs careful evaluation within the first hours of onset. Blood cultures, Gram stain, and urinary detection of antigens should be done.

Given the relevance of pulmonary infection due to PRSP (throughout the world) and Legionella spp. (some countries), we believe that antibiotic treatment must cover these microorganism in critically ill patients when SCAP etiology is unknown. In addition, antibiotic coverage for Pseudomonas might be necessary in intubated patients with SCAP even if risk factors are not present. Early recognition of severity, adequate empirical antibiotic therapy, and improvements in adjunctive therapy for multiorgan dysfunction are key elements for improving outcome.

We believe that cefotaxime/ceftriaxone should be the cornerstone of therapy in those patients with a high prevalence of PRSP in combination with macrolide, whereas new fluoroquinolones should be reserved for allergic patients or documented S. pneumoniae with MIC of more than $4 \mu \mathrm{g} / \mathrm{mL}$, which is isolated very infrequently.

\section{Acknowledgment}

We are indebted to María Bodí, MD for critical review of the manuscript.

\section{References}

1. Barlett JG, Dowell SF, Mandell LA, et al. Practice guidelines for the management of community-acquired pneumonia in adults. Clin Infect Dis 2000;31:347-82.

2. Hirani NA, Macfarlane JT. Impact of management guidelines on the outcome of severe community acquired pneumonia. Thorax 1997;52:17-21.

3. Almirall J, Bolibar I, Vidal J, et al. Epidemiology of community-acquired pneumonia in adults: a population-based study. Eur Respir J 2000;15:757-63.

4. Jokinen $\mathrm{C}$, Heiskanen L, Juvonen $\mathrm{H}$, et al. Incidence of community-acquired pneumonia in the population of four municipalities in Eastern Finland. Am J Epidemiol 1993;137:977-88.

5. Lave JR, Lin CJ, Fine MJ, et al. The cost of treating patients with community-acquired pneumonia. Semin Respir Crit Care Med 1999;20:189-97.

6. Gallego M, Rello J. Severe community-acquired pneumonia. In: Rello J, Vallés J, and 
Kollef M, eds. Critical care infectious diseases textbook. Dordrecht: Kluwer Academic Publishers, 2001:37:611-23.

7. American Thoracic Society. Guidelines for the initial management of adults with community-acquired pneumonia: diagnosis, assessment of severity and initial antimicrobial therapy. Am Rev Respir Dis 1993;148:1418-26.

8. European Study on Community-Acquired Pneumonia (ESOCAP) Committee. Guidelines for management of adult community-acquired lower respiratory tract infections. Eur Respir J 1998,11:986-98.

9. BTS guidelines for the management of community-acquired pneumonia in adults. Thorax 2001;56[Suppl 4]S1-S64.

10. Ewing S, Ruiz M, Mensa J, et al. Severe community-acquired pneumonia: assessment of severity criteria. Am J Respir Crit Care Med 1998,158:1102-8.

11. Research Committee of British Thoracic Society and the Health Laboratory Service. Community-acquired pneumonia in adults in British hospitals in 1982-83: a survey of aetiology, mortality, prognostic factors and outcome. Q J Med 1987,62:195-201.

12. Arnold FW, Ramirez JA, McDonald LC, et al. Hospitalization for community-acquired pneumonia. Chest 2003;124:121-4.

13. Fine MJ, Auble TE, Yealy DM, et al. A predictive rule to identify low-risk patients with community-acquired pneumonia. N Engl J Med 1997;336:243-50.

14. Rosón B, Carratalá J, Dorca J, et al. Etiology, reasons for hospitalization, risk classes, and outcome of community-acquired pneumonia in patients hospitalized on the basis of conventional admission criteria. Clin Infect Dis 2001;33:158-65.

15. Marras TK, Gutierrez C, Chan C. Applying a prediction rule to identify low-risk patients with community-acquired pneumonia. Chest 2000;118:1339-43.

16. Zalacain R, Torres A, Celis R, et al. Community-acquired pneumonia in the elderly: Spanish multicentre study. Eur Respir J 2003;21:294-302.

17. El-Ebiary M, Sarmiento X, Torres A, et al. Prognostic factors of severe Legionella pneumonia requiring admission to ICU. Am J Respir Crit Care Med 1997;156:146772.

18. Roig J, Rello J. Legionnaires' disease: a rational approach to therapy. J Antimicrob Chemother 2003;51:1119-29.

19. Roig J, Sabria M, Pedro-Botet ML. Legionella spp: community acquired and nosocomial infections. Curr Opin Infect Dis 2003;16:145-51.

20. Lopardo G, Sturba E, Martínez ML, et al. Detection of acute infection due to Legionella pneumophila in patients with community acquired pneumonia in Buenos Aires city. Medicine (B. Aires) 2002;62:145-8.

21. Cabello H, Cortes C, Ruiz M, et al. Community acquired pneumonia. Report of 8 cases of severe pneumonia by serogroup 1 Legionella pneumophila in Chile. Rev Med Chil 2002;130:309-13.

22. Woodhead M. Community-acquired pneumonia in Europe: causative pathogens and resistance patterns. Eur Respir J 2002;20[Suppl 36]:20S-27S.

23. Pachón J, Prado MD, Capote F, et al. Severe community acquired pneumonia: etiology, prognosis and treatment. Am Rev Respir Dis 1990;142:369-73.

24. Rello J, Quintana E, Ausina V, et al. A three-year study of severe community acquired pneumonia with emphasis in outcome. Chest 1993;103:232-5.

25. Moine $P$, Vercken JB, Chevret $S$, et al. Severe community-acquired pneumonia: etiology, epidemiology, and prognosis factors. Chest 1994;105:1487-95.

26. Leroy O, Santré C, Beuscart C, et al. A five-year study of severe community-acquired 
pneumonia with emphasis on prognosis in patients admitted to an intensive care unit. Intensive Care Med 1995;21:24-8.

27. Rello J, Rodríguez R, Jubert $P$, et al. Severe community-acquired pneumonia in the elderly: epidemiology and prognosis. Clin Infect Dis 1996;23:723-8.

28. Wattanathum A, Chaoprasong C, Nunthapisud $P$, et al. Community-acquired pneumonia in Southeast Asia. The microbial differences between ambulatory and hospitalized patients. Chest 2003;123:1512-9.

29. Rello J, Bodi M, Mariscal D, et al. Microbiological testing and outcome of patients with severe community-acquired pneumonia. Chest 2003;123:174-80.

30. Jasovich A, Soutric J, Morera G, et al. Efficacy of amoxicillin-sulbactam, given twice a day for the treatment of community-acquired pneumonia. A clinical trial based on a pharmacodynamic model. J Chemother 2002;14:591-6.

31. Soca A, Bazet C, Battistesa S, et al. Severe community-acquired pneumonia. Pac Crit 1999;12:96-107.

32. Bantar C, Bavestrello L, Crucio D, et al. Acute community-acquired pneumonia in adults. Guidelines for initial antimicrobial therapy based on local evidence from a South American Working Group (Conesur). J Chemother 2002;14[Suppl 4]:S1-S22.

33. Marrie TJ, Peeling RW, Reid T, et al. Chlamydia species as a cause of communityacquired pneumonia in Canada. Eur Respir J 2003;21:779-84.

34. Menéndez R, Córdoba J, de la Cuadra P, et al. Value of the polymerase chain reaction assay in noninvasive respiratory samples for diagnosis of community-acquired pneumonia. Am J Respir Crit Care Med 1999;159:1868-3.

35. Rello J, Paiva A, Dias CS. Current dilemmas in the management of adults with severe community-acquired pneumonia. In: Vincent JL, ed. Yearbook of intensive care and emergency medicine. New York: Springer-Verlag, 2003:162-74.

36. Ewing S, Bauer T, Hasper E, et al. Value of routine microbial investigation in community-acquired pneumonia treated in a tertiary care centre. Respiration 1991;63:164-9.

37. Waterer GW, Wunderink RG. The influence of the severity of community-acquired pneumonia on the usefulness of blood cultures. Respir Med 2001;95:78-82.

38. Leeper KV, Torres A. Community-acquired pneumonia in the intensive care unit. Clin Chest Med 1995;16:155-71.

39. Rosón B, Guidol F. Utility of Gram stain and sputum culture in the management of community-acquired pneumonia. Clin Pulm Med 2003;10:1-5.

40. Ewing S, Torres A. Severe community-acquired pneumonia. Clin Chest Med 1999;20:575-80.

41. Mandell LA, Marrie TJ, Grossman RF, et al. Canadian guidelines for the initial management of community-acquired pneumonia: an evidence-based update by Canadian Infectious Diseases Society and Canadian Thoracic Society. Clin Infect Dis 2000;31:383421.

42. Sanyal S, Smith PR, Saha AC, et al. Initial microbiologic studies did not affect outcome in adults hospitalized with community-acquired pneumonia. Am J Respir Crit Care Med 1999,160:346-8.

43. Campbell SG, Marrie TJ, Anstey R, et al. The contribution of blood cultures to the clinical management of adult patients admitted to the hospital with community-acquired pneumonia. A prospective observational study. Chest 2003;123:1142-50.

44. Vallés J, Rello J, Ochagavía A, et al. Community-acquired bloodstream infection in critically ill adult patients. Impact of shock and inappropriate antibiotic therapy on survival. Chest 2003;123:1615-24. 
45. Rello J, Gallego M, Mariscal D, et al. The value of routine microbial investigation in ventilator-associated pneumonia. Am J Respir Crit Care Med 1997;156:196-200.

46. American Thoracic Society. ATS guidelines for the management of adults with community-acquired pneumonia: diagnosis, assessment of severity. Antimicrobial therapy and prevention. Am J Respir Crit Care Med 2001;163:1730-54.

47. Feinsilver SH, Fein AM, Niederman NS, et al. Utility of fiberoptic bronchoscopy in non-resolving pneumonia. Chest 1990;98:1322-6.

48. Fine MJ, Smith MA, Carson CA, et al. Prognosis and outcomes of patients with community acquired pneumonia: a meta-analysis. JAMA 1996;276:134-41.

49. Marrie TJ. Epidemiology of community acquired pneumonia in the elderly. Semin Respir Infect 1990;5:260-8.

50. Riquelme R, Torres A, El-Ebiary M, et al. Community acquired pneumonia in the elderly. A multivariate analysis of risks and prognostic factors. Am J Respir Crit Care Med 1996;154:1450-5.

51. Fernández-Sabé N, Carratalà J, Rosón B, et al. Community-acquired pneumonia in very elderly patients: causative organisms, clinical characteristics and outcomes. Medicine (Baltimore) 2003;82:159-69.

52. Lim WS, van der Eerden MM, Laing R, et al. Defining community-acquired pneumonia severity on presentation to hospital: an international derivation and validation study. Thorax 2003;58:377-82.

53. Torres A, Dorca J, Zalacaín R, et al. Community-acquired pneumonia in chronic obstructive pulmonary disease. A Spanish multicenter study. Am J Respir Crit Care Med 1996;154:1456-61.

54. Seneff MG, Wagner DP, Wagner RD, et al. Hospital and 1-year survival of patients admitted to intensive care units with acute exacerbation of chronic obstructive pulmonary disease. JAMA 1995;274:1852-7.

55. Torres A, Serra-Batlles J, Ferrer A, et al. Severe community acquired pneumonia: epidemiology and prognostic factors. Am Rev Respir Dis 1991;114:312-5.

56. Meehan TP, Fine MJ, Krumholz HM, et al. Quality of care, process, and outcome in elderly patients with pneumonia. JAMA 1997;278:2080-4.

57. Saldías Peñafiel F, O’Brien Solar A, Gederlini Gollerino A, et al. Community-acquired pneumonia requiring hospitalization in immunocompetent elderly patients: clinical features, prognostic factors and treatment. Arch Bronconeumol 2003;39:333-40.

58. Bantar C, Bavestrello L, Curcio D, et al. Acute community-acquired pneumonia in adults: guidelines for initial antimicrobial therapy based on local evidence from a South American Working Group (ConsenSur). J Chemother 2002;14:1-22.

59. Rello J, Catalán M, Díaz E, et al. Associations between empirical antimicrobial therapy at the hospital and mortality in patients with severe community-acquired pneumonia. Intensive Care Med 2002;28:1030-5.

60. Luna C, Famiglietti A, Absi R, et al. Community-acquired pneumonia: etiology, epidemiology and outcome at a teaching hospital in Argentina. Chest 2000;118:1344-54.

61. Lujan M, Gallego M, Fontanals D, et al. Association of discordant antibiotic therapy and mortality in adults with bacteremic pneumococcal community-acquired pneumonia. Crit Care Med 2004;32(3):625-31.

62. Yu VL, Chiou CCC, Feldman C, et al. An international prospective study of pneumococcal bacteremia: correlation with in vitro resistance, antibiotic administered and clinical outcome. Clin Infect Dis 2003;37:230-37.

63. Orranti AR, Silva H, Pontani D, et al. El grupo de estudio Artemis para America Latina: el proyecto Artemis, un estudio sobre la actividad de algunos ATB de uso común para 
el tratamiento de las infecciones en diez países de Latinoamerica. Rev Panam Infectolog 1998;2:68-75.

64. Guzmán-Blanco M, Casellas JM, Sader HS, et al. Bacterial resistance to antimicrobial agents in Latin America. The giant is awakening. Infect Dis Clin North Am 2000;14:6781.

65. Vidaur L, Rodríguez A, Rello J. ATB therapy for sepsis, severe sepsis and septic shock.:A patient-based approach inspired on "The Tarragona Strategy." In: Yearbook of intensive care and emergency medicine. New York: Springer-Verlag, (in press).

66. Vincent JL. Hemodynamic support in septic shock. Intensive Care Med 2001;27:S80 S92.

67. Neviere R, Mathieu D, Chagnon JL, et al. The contrasting effects of dobutamine and dopamine on gastric mucosal perfusion in septic patients. Am J Respir Crit Care Med 1996;154:1684-8.

68. Marik PE, Mohedin J. The contrasting effects of dopamine and norepinephrine on systemic and splanchnic oxygen utilization in hyperdynamic sepsis. JAMA 1994;272:1354-7.

69. Ronco JJ, Fenwick JC, Tweeddale MG, et al. Identification of the critical oxygen delivery for anaerobic metabolism in critically ill septic and nonseptic humans. JAMA 1993;270:1724-30.

70. Kellum JA, Kramer DJ, Lee K, et al. Release of lactate by lung in acute lung injury. Chest 1997;111:1301-5.

71. Gutierrez G, Palizas F, Doglio GR, et al. Gastric intramucosal pH as a therapeutic index of tissue oxygenation in critically ill patients. Lancet 1992;339:195-9.

72. Girou E. Prevention of nosocomial infections in acute respiratory failure patients. Eur Respir J 2003;42[Suppl]:72-6.

73. Domenighetti G, Gayer R, Gentilini R. Noninvasive pressure support ventilation in non-COPD patients with acute cardiogenic pulmonary edema and severe community-acquired pneumonia: acute effects and outcome. Intensive Care Med 2002;28:1226-32.

74. Confalonieri M, Potena A, Carbone G, et al. Acute respiratory failure in patients with severe community-acquired pneumonia: a prospective randomized evaluation of noninvasive ventilation. Am J Respir Crit Care Med 1999;160:1585-91.

75. Jolliet $\mathrm{P}$, Abajo B, Pasquina $\mathrm{P}$, et al. Non-invasive pressure support ventilation in severe community-acquired pneumonia. Intensive Care Med 2001;27:812-21.

76. The Acute Respiratory Distress Syndrome Network. Ventilation with lower tidal volumes as compared with traditional tidal volumes for acute lung injury and the acute respiratory distress syndrome. N Engl J Med 2000;342:1301. 